\title{
Self-Powered Implantable Skin-Like Glucometer for Real-Time Detection of Blood Glucose Level In Vivo
}

\author{
Wanglinhan Zhang ${ }^{1} \cdot$ Linlin Zhang ${ }^{1} \cdot$ Huiling Gao $^{2} \cdot$ Wenyan Yang ${ }^{1} \cdot$ Shuai Wang $^{1} \cdot$ Lili Xing $^{1} \cdot$ Xinyu Xue $^{1}$
}

Received: 24 October 2017/Accepted: 16 December 2017/Published online: 4 January 2018

(C) The Author(s) 2018. This article is an open access publication

\section{Highlights}

- Self-powered implantable skin-like glucometer for real-time detection of blood glucose level in vivo was fabricated.

- The distinct interdigitated electrode ensures piezo-potential of individual nanowires in the same direction. Piezobiosensing process does not require an external power supply. And the piezoelectric-enzyme-reaction coupling effect is proposed.

- The device can run well in live mouse and detect in real time the glucose level.

\begin{abstract}
Implantable bioelectronics for analyzing physiological biomarkers has recently been recognized as a promising technique in medical treatment or diagnostics. In this study, we developed a self-powered implantable skinlike glucometer for real-time detection of blood glucose level in vivo. Based on the piezo-enzymatic-reaction coupling effect of GOx@ $\mathrm{ZnO}$ nanowire, the device under an applied deformation can actively output piezoelectric signal containing the glucose-detecting information. No external electricity power source or battery is needed for this device, and the outputting piezoelectric voltage acts as
\end{abstract}

Electronic supplementary material The online version of this article (https://doi.org/10.1007/s40820-017-0185-x) contains supplementary material, which is available to authorized users.

Wanglinhan Zhang and Linlin Zhang have contributed equally to this work.

Shuai Wang

s_wang@mail.neu.edu.cn

$凶$ Xinyu Xue

xuexinyu@mail.neu.edu.cn

1 College of Sciences, Northeastern University, Shenyang 110004, People's Republic of China

2 College of Life and Health Sciences, Northeastern University, Shenyang 110004, People's Republic of China

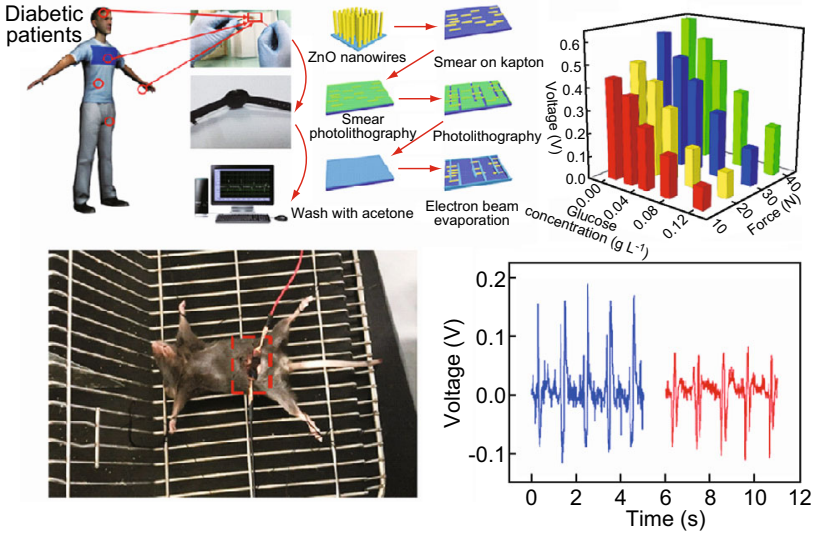

both the biosensing signal and electricity power. A practical application of the skin-like glucometer implanted in mouse body for detecting blood glucose level has been simply demonstrated. These results provide a new technique path for diabetes prophylaxis and treatment.

Keywords Diabetes · Biosensor - Electronic-skin - Selfpowered $\cdot$ Glucose detection $\cdot$ Implantable electronics 


\section{Introduction}

Diabetes mellitus (DMs), a group of metabolic diseases, in which there are high blood glucose levels over a prolonged period, is among the series of problems challenging the human health. If left untreated, diabetes can cause a series of complications, such as blindness, diabetic foot, renal failure, heart disease, and stroke. Since diabetes may exist without clinical manifestations at the early stage, symptomatic cases may be difficult to detect. Continuous blood glucose monitoring in daily life is necessary for prophylaxis and early treatment of diabetes [1, 2]. At present, the most commonly utilized approach in medical field is blood test. Although the test of blood sample in vitro can directly monitor blood glucose with great precision, some shortcomings still exist. One is the difficulty of realizing real-time monitoring, and the other is that a blood test needs too many steps, including blood sampling and chemical analysis [3].

With the rapid development of semiconductor industry and information technology, wearable electronics (e.g., electronic skin) with various functions have attracted a wide range of attention. Recently, the wearable skin-like biological sensors have emerged as effective noninvasive transducers of human physiological signals in healthcare [4-11]. However, there are still lots of challenges of skinlike biosensors for implanting in human body. First of all, as the skin-like biosensor is implanted inside human body, the material must be flexible and soft, and the biosensing functions must have clinical significance $[12,13]$. On the other hand, energy consumption is a bottleneck problem for the skin-like biosensors to be used inside the body in large scale and long term. Although common electricity power sources (e.g., batteries) have high energy density, they are not suitable for integrating in the skin-like biosensing systems. The rigid shell of the battery and leakage risk are opposite to the implanting requirements, and the charging and replacing processes of the battery are inconvenient in the inner body environment. Our previous work shows that the piezo-enzymatic-reaction coupling effect of GOx@ZnO (GOx: glucose oxidase) nanowires can realize detecting glucose concentration without battery, but the device structure and implanting operation need to be developed [14].

The World Health Organization (WHO) predicts that there will be 300 million people having diabetes by the year 2025 (Fig. 1a). The continuous monitoring of blood glucose concentration is strongly requested [15-18]. Here,

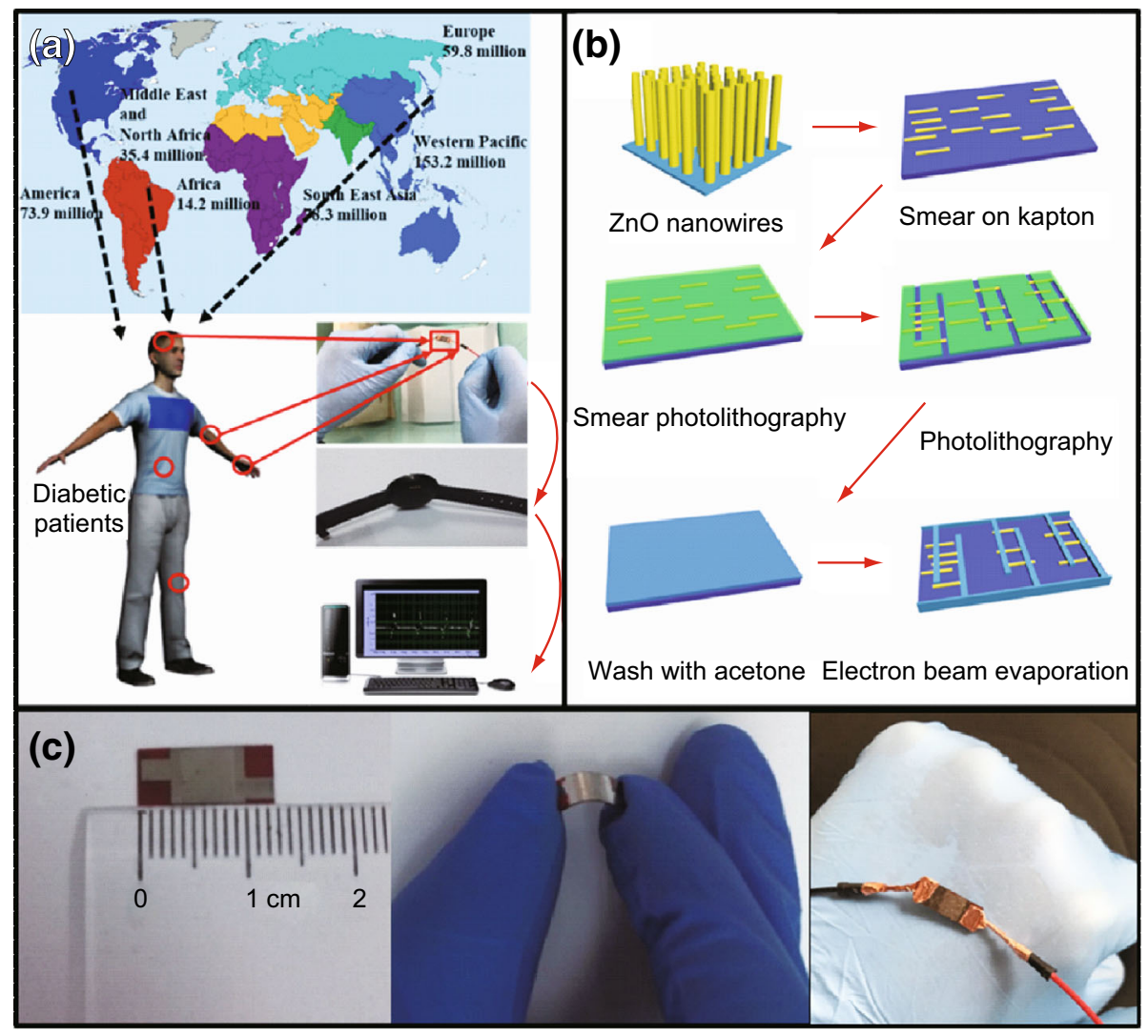

Fig. 1 Potential application, device architecture, material system, and experimental design of self-powered implantable skin-like glucometer. a Potential application. b Device architecture, material system, and fabrication procedure. c Optical images of the device 
a self-powered implantable skin-like glucometer for realtime detection of blood glucose level has been realized based on the piezo-enzymatic-reaction coupling process of horizontally aligned GOx@ZnO nanowire arrays [19-22]. The device can actively output piezoelectric voltage under applied deformation, and the outputting piezoelectric voltage contains the information of glucose concentration inside the body [23-26]. The outputting piezoelectric voltage can act as not only the electricity power for driving the device but also the biosensing signal. In this process, no external electricity power source or battery is needed [27-29]. A practical application of the device implanted in mouse body for detecting its blood glucose level without any external electricity power has been simply demonstrated. Our results could provoke a possible new research field of self-powered/real-time diabetes monitoring and diagnosis.

\section{Experimental}

\subsection{Fabrication of the Self-Powered Implantable Skin-Like Glucometer}

All the analytical-grade chemical reagents for synthesizing GOx@ZnO nanowire arrays were supplied by Sinopharm Chemical Reagent Co. Ltd.

A seed-assisted hydrothermal method was used to align $\mathrm{ZnO}$ nanowire arrays vertically on the Ti substrate [30-32]. Firstly, the Ti substrate (thickness of $100 \mu \mathrm{m}$ ) was washed for several times with alcohol and DI water to remove surface impurities, and dried in an oven at $60^{\circ} \mathrm{C}$. $\mathrm{Zn}\left(\mathrm{CH}_{3} \mathrm{COO}_{2}\right)_{2} \cdot \mathrm{H}_{2} \mathrm{O}$ solution $(10 \mathrm{mM}$ in ethanol) was dropped onto the pre-cleaned Ti substrate and blown dry with nitrogen gas. This process was repeated for several times. Secondly, in order to produce the seed layer, the $\mathrm{Ti}$ substrate was conducted with an annealing treatment at $350{ }^{\circ} \mathrm{C}$ for $20 \mathrm{~min}$. Thirdly, $0.416 \mathrm{~g}$ of $\mathrm{Zn}\left(\mathrm{NO}_{3}\right)_{2} \cdot 6 \mathrm{H}_{2} \mathrm{O}$ was dissolved in $38 \mathrm{~mL}$ of DI water and stirred for $10 \mathrm{~min}$ at room temperature. Two milliliters of $\mathrm{NH}_{3} \cdot \mathrm{H}_{2} \mathrm{O}$ was added into the solution under continuous stirring, and the Ti substrate was immersed in the solution. After that, the reaction beaker was sealed and maintained at $90{ }^{\circ} \mathrm{C}$ for $24 \mathrm{~h}$. Finally, Ti substrate was taken out, rinsed with ethanol/DI water, and dried at $60{ }^{\circ} \mathrm{C}$.

As the flexible substrate for supporting the device, a Kapton board was cleaned with alcohol and DI water for several times and dried at $60{ }^{\circ} \mathrm{C}$. The horizontally aligned $\mathrm{ZnO}$ nanowire arrays (overwhelmed down on $\mathrm{Ti}$ substrate) were carefully transferred to the Kapton substrate, while ensuring that all the nanowires were in the uniform direction. The Kapton substrate with $\mathrm{ZnO}$ nanowires was patterned by photolithography using positive photoresist. The
$200 \mathrm{~nm}$-thick layer of $\mathrm{Ti}$ as electrode was subsequently deposited by electron beam evaporation at room temperature. In order to remove the abundant $\mathrm{Ti}$ and photoresist, the Kapton substrate was immersed in an organic solvent, rinsed with deionized water, and dried at $60{ }^{\circ} \mathrm{C}$. Finally, $\mathrm{ZnO}$ nanowires were modified with GOx (supplied by Sigma Chemical Co. St. Louis, MO, USA). $0.5 \mathrm{~mL}$ of the configured GOx aqueous solution $\left(10 \mathrm{~g} \mathrm{~L}^{-1}\right)$ was slowly dropped onto the surface of $\mathrm{ZnO}$ nanowires. The device was placed in a dry and ventilated place for 2-3 $\mathrm{h}$ [33]. This process was repeated four times to complete the attachment of GOx [34]. Finally, each device should be covered with $20 \mathrm{mg}$ GOx on $0.4 \mathrm{~cm}^{2}$ area. In the previous work, $\mathrm{ZnO}$ has proven to be nontoxic and can work well in the human body environment, as well as the substrate of Kapton and the electrode material of $\mathrm{Ti}$ [35-39]. Thus, the whole material system in our device is nontoxic and biocompatible.

\subsection{Characterization and Measurement}

The crystal phase of $\mathrm{ZnO}$ nanowire arrays was characterized by X-ray diffraction (XRD, D/max $2550 \mathrm{~V}, \mathrm{Cu} \mathrm{K} \alpha$ radiation). The morphology and microstructure of the nanowires and the skin-like glucometer device were investigated by a scanning electron microscope (SEM, JEOL JSM-6700F) and transmission electron microscope (TEM, JEOL JEM-2010).

The skin-like glucometer was completely submerged in the aqueous solution of glucose. A programmed motor which could provide a constant force for simulating body motion was used to drive the device with a fixed frequency. The force was applied on the center of the device (the supporters were on the sides), and the device can be bent. A force sensor was used to measure the magnitude of the force. The outputting piezoelectric voltage was measured using a low-noise preamplifier (Model SR560, Stanford Research Systems).

\subsection{Experiment In Vivo}

The skin-like glucometer was implanted into the mouse body, and the surgery process was as follows. The mouse was injected with anesthetic for the following surgery. The device was implanted into the mouse's abdomen under the skin, where the biosensor unit can directly contact with the mouse blood. The edges of the device were connected with two electrode poles to receive the outputting piezoelectric voltage generated by the device [40]. After that, we slowly injected $5 \mathrm{~mL}$ of glucose solution $\left(0.045 \mathrm{~g} \mathrm{~L}^{-1}\right)$ into the mouse abdomen using a medical syringe within about $5 \mathrm{~s}$. All of the procedures in this study conformed to the regulations for the administration of affairs concerning 
experimental animals and were approved by Northeastern University.

\section{Results and Discussion}

Figure 1a shows the experimental design of this work. Figure 1b schematically illustrates the material system and fabrication procedure of self-powered implantable skin-like glucometer [40, 41]. The device is assembled by positioning parallel $\mathrm{ZnO}$ nanowires (with uniform direction) onto the $\mathrm{Ti}$ interdigitated electrodes on a soft Kapton substrate. To ensure the outputting piezoelectric voltage, the distance between neighboring electrode couples is $20 \mu \mathrm{m}$, larger than the length of $\mathrm{ZnO}$ nanowire $(\sim 12 \mu \mathrm{m})$ [42]. Figure 1c shows the optical images of the device. The thin device is $0.4 \times 1.3 \mathrm{~cm}^{2}$ in size and can be easily bended. The thickness of the Kapton film is $250 \mu \mathrm{m}$, and the overall thickness of the device is less than $260 \mu \mathrm{m}$. Compared with our previous work [14], the device structure and application are greatly improved in this study. The new substrate is more flexible and stretchable. The nanowires are horizontally aligned on the substrate instead of vertical arrangement, which can facilitate the biosensing process. The new device is very small and suitable for embedding into the organism and can be implanted into mouse body to detect glucose concentration in vivo.

The morphology and structure of $\mathrm{ZnO}$ nanowire arrays and self-powered implantable skin-like glucometer are shown in Fig. 2. Figure 2a shows the SEM image of asgrown $\mathrm{ZnO}$ nanowire arrays on $\mathrm{Ti}$ substrate on the side view, showing that $\mathrm{ZnO}$ nanowires are vertically aligned on the substrate. The average length of the nanowires is about $12 \mu \mathrm{m}$. Figure 2b, c shows the top-view SEM images of as-grown $\mathrm{ZnO}$ nanowire arrays, showing that $\mathrm{ZnO}$ nanowires have a uniform distribution on the substrate with the same growth direction and the average diameter of the nanowires is about $160 \mathrm{~nm}$. Figure 2d shows the SEM image of horizontally aligned $\mathrm{ZnO}$ nanowire arrays (overwhelmed in one direction) before smearing on Kapton substrate [43]. The same growth direction of the overwhelmed nanowire arrays ensures that the crystallographic orientations of the nanowires in the following device are aligned along the same direction. Consequently, the

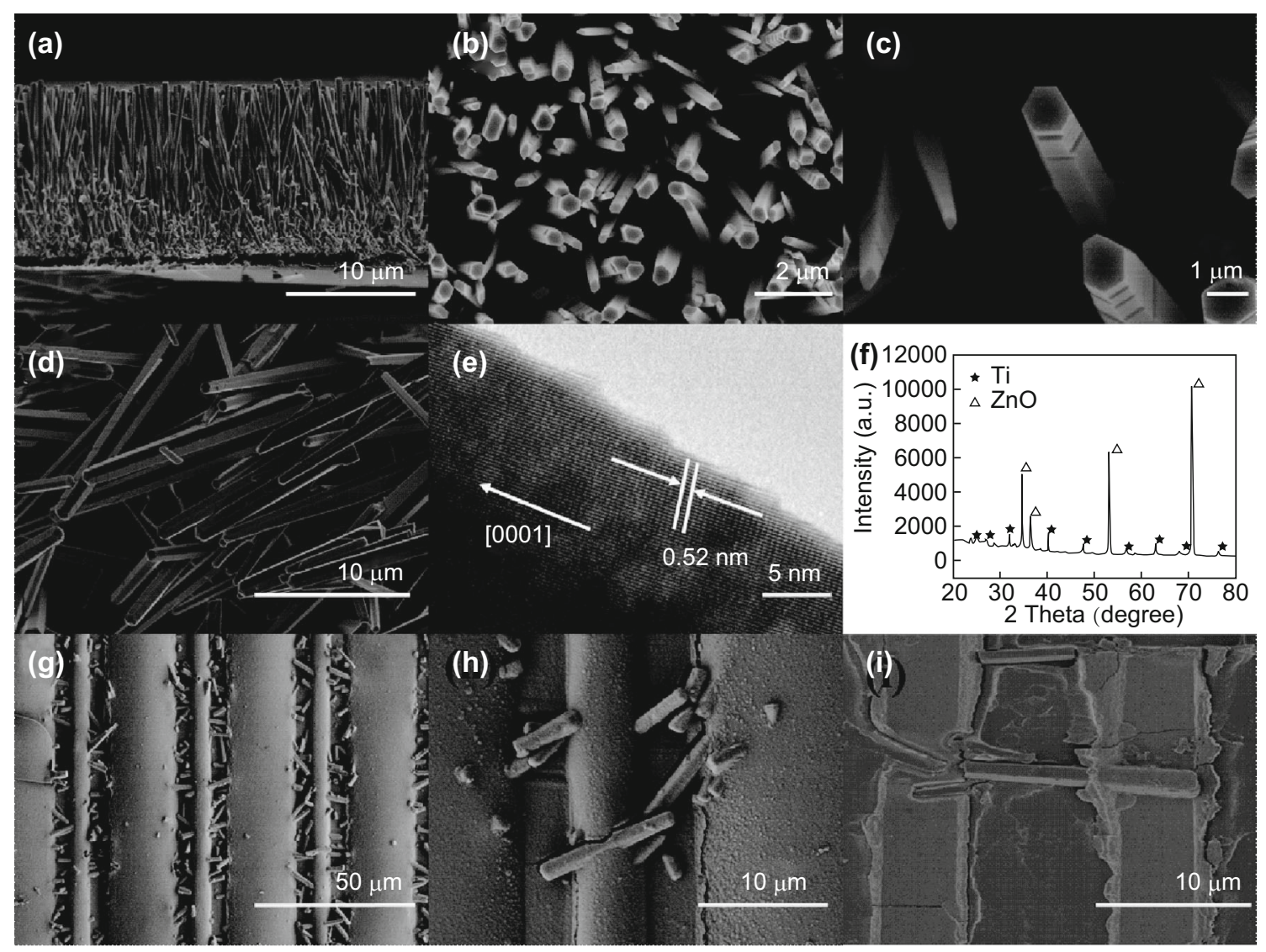

Fig. 2 a SEM image of as-grown ZnO nanowire arrays on Ti substrate on the side view. b, c Top-view SEM images of as-grown ZnO nanowire arrays. d SEM image of ZnO horizontally aligned nanowire arrays (overwhelmed in one direction) before smearing on Kapton substrate. e Highresolution TEM image of one single $\mathrm{ZnO}$ nanowire. $\mathbf{f}$ XRD pattern of $\mathrm{ZnO}$ nanowires. $\mathbf{g}$ Top-view SEM image of the device (before washing with acetone). $\mathbf{h}$, $\mathbf{i}$ Enlarged view of the device 
(a)

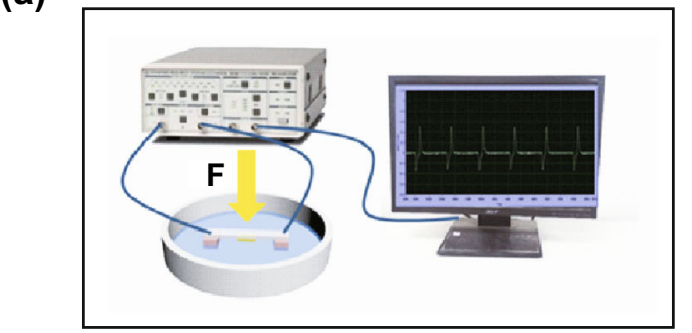

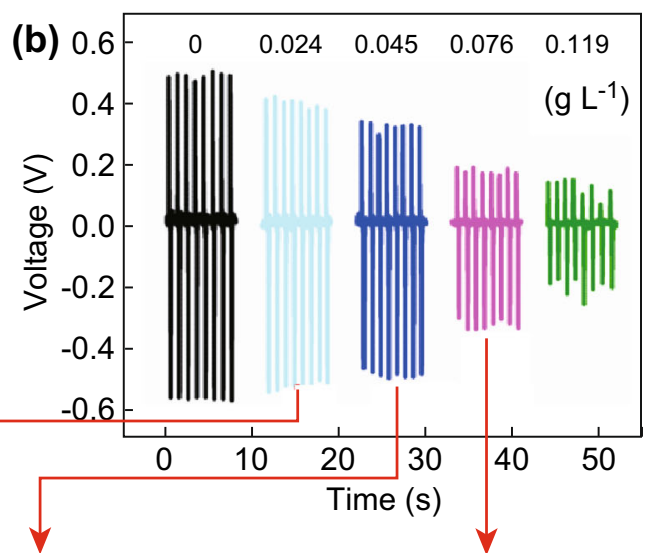

(c)
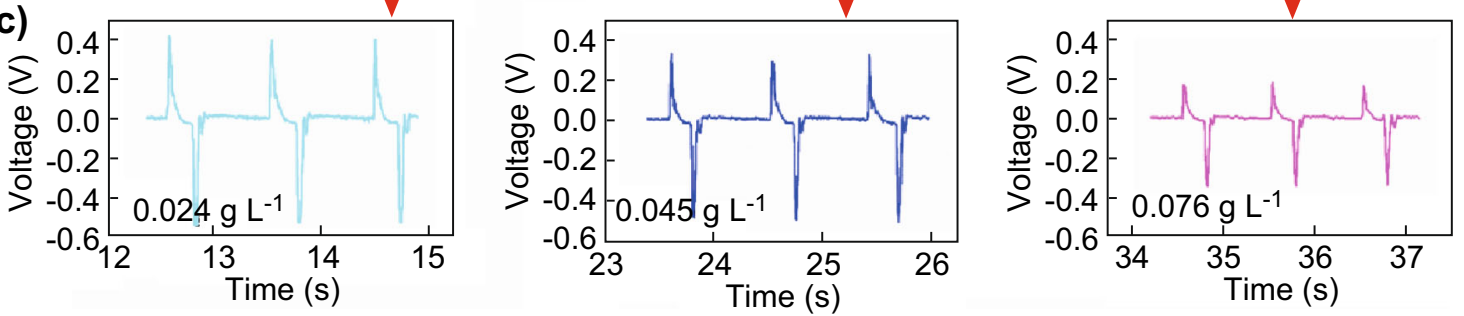

(d)

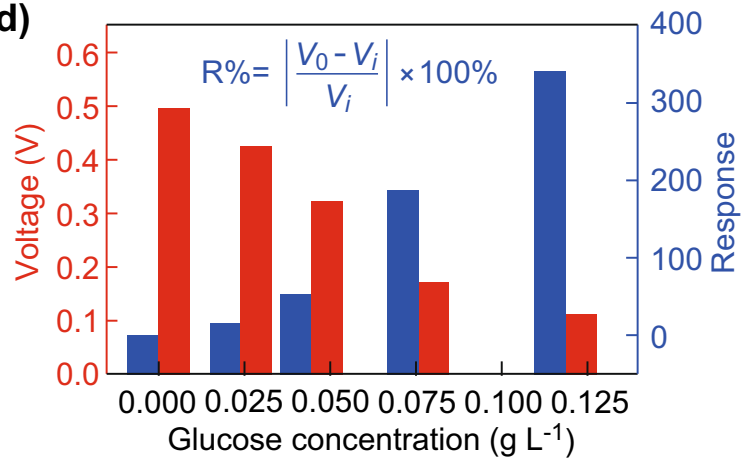

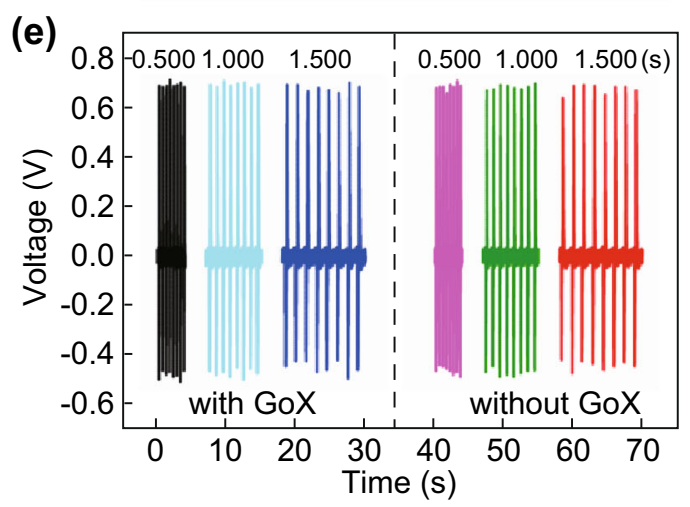

Fig. 3 a The measurement system. b The outputting piezoelectric voltage of self-powered implantable skin-like glucometer in different concentrations of glucose solution. The applied force is $21 \mathrm{~N}$. c The enlarged views of the outputting piezoelectric voltage against $0.024,0.45$, and $0.076 \mathrm{~g} \mathrm{~L}^{-1}$ glucose solution. d The outputting piezoelectric voltage and the response of the device against different glucose solution concentration. $\mathbf{e}$ The piezoelectric voltage of the skin-like glucometer with and without GOx modification in air with different force frequencies

polarities of the induced piezo-potential of individual nanowires are also in the same direction, leading to a macroscopic potential contributed constructively by all of $\mathrm{ZnO}$ nanowires. Figure 2e shows the high-resolution TEM image of one single $\mathrm{ZnO}$ nanowire. It can be observed that the lattice spacing is $0.52 \mathrm{~nm}$, consistent with (001) crystal plane of wurtzite structural $\mathrm{ZnO}[44,45]$. The XRD pattern of $\mathrm{ZnO}$ nanowires is shown in Fig. $2 \mathrm{f}$, and the sharp diffraction peaks indicate good crystalline quality. All the diffraction peaks can be indexed to Ti (JCPDS card no. 44-1294) and the hexagonal wurtzite structure of $\mathrm{ZnO}$ (JCPDS card no. 36-1451). The top-view SEM image of the device (before washing with acetone) and the enlarged view are shown in Fig. $2 \mathrm{~g}$, h, respectively. $\mathrm{ZnO}$ nanowires locate across $\mathrm{Ti}$ interdigitated electrodes. Figure $2 \mathrm{i}$ shows that after removing the photoresist with acetone one single
$\mathrm{ZnO}$ nanowire bridges the two electrodes in one electrode couple.

Figure 3 shows the piezoelectric-biosensing performance of self-powered implantable skin-like glucometer. The device is completely immersed in the aqueous solution of glucose, as shown in Fig. 3a [46]. Under an applied deformation, the device (being bended) can transfer the mechanical energy to piezoelectric voltage that carries the information of glucose concentration. No other electric power is needed during this process, and the outputting piezoelectric voltages can act as both the power source and the biosensing signal. Figure $3 \mathrm{~b}$ shows that the outputting piezoelectric voltage of the device decreases with increasing glucose concentration. As the concentrations of glucose are $0,0.024,0.045,0.076$, and $0.119 \mathrm{~g} \mathrm{~L}^{-1}$, the voltage of the device is about $0.49,0.42,0.32,0.17$, and 

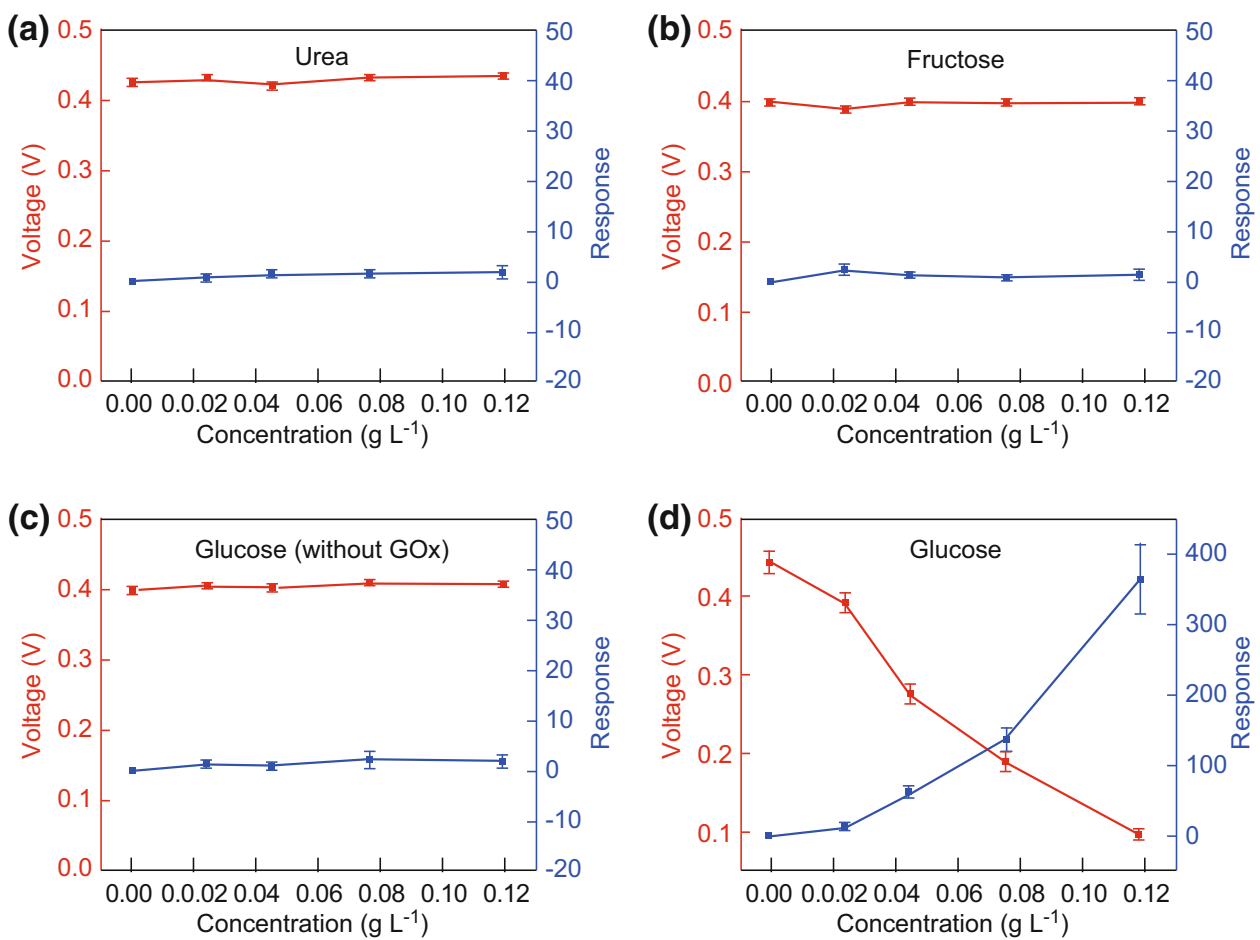

Fig. 4 a, b The outputting piezoelectric voltage and response of the self-powered implantable skin-like glucometer against urea and fructose. c The outputting piezoelectric voltage and response of the device without GOx modification against glucose. d The outputting piezoelectric voltage and response of the device with GOx modification against glucose

$0.11 \mathrm{~V}$, respectively. The enlarged views of the outputting piezoelectric voltage against $0.024,0.045$, and $0.076 \mathrm{~g} \mathrm{~L}^{-1}$ are shown in Fig. 3c. These results indicate that the device can monitor the glucose concentration [47, 48].

The response of the device can be calculated from Eq. 1 [49]:

$R \%=\left|\frac{V_{0}-V_{i}}{V_{i}}\right| \times 100 \%$

where $V_{0}$ and $V_{\mathrm{i}}$ are the outputting piezoelectric voltage of the device in pure water and glucose aqueous solution, respectively. Figure $3 \mathrm{~d}$ shows that the response of the device against glucose concentrations of $0.024,0.045$, 0.076 , and $0.119 \mathrm{~g} \mathrm{~L}^{-1}$ is $16,53,188$, and 340 , respectively. Obviously with the increasing concentration of glucose solution, the outputting piezoelectric voltage is reduced. On the contrary, the response increases with increasing glucose concentration. This device can sensitively detect glucose concentration, and no external electricity power is needed in the piezo-biosensing process. The outputting piezoelectric voltage is linearly dependent on glucose concentration (0.024-0.119 $\left.\mathrm{g} \mathrm{L}^{-1}\right)$, as shown in Fig. S4. The limit of detection (LOD; the signal-to-noise ratio is $3: 1$ ) can be calculated to be about $0.019 \mathrm{~g} \mathrm{~L}^{-1}$ [50]. Figure $3 \mathrm{e}$ exhibits the piezoelectric voltage of $\mathrm{ZnO}$ nanowires with and without GOx modification in air with different force frequencies. It can be seen that the outputting piezoelectric voltage arises from the piezoelectric effect of $\mathrm{ZnO}$ nanowires.

When the self-powered implantable skin-like glucometer is practically used inside a human body, the various kinds of electrolytes and metabolites in body fluid may influence the biosensing accuracy [51]. Therefore, selectivity is an important parameter of the device to detect specific target analytes. Figure 4 shows the selectivity test of the device against three kinds of common ingredients in the body fluid (glucose, fructose, and urea) [52, 53]. The response of the device against fructose and urea is very small (Fig. 4a, b). Figure 4c, d shows that the change in outputting piezoelectric voltage is due to the reactions between glucose and oxidase, rather than the influence of water. The results suggest that the device has a good selectivity for detecting glucose. The influence caused by other electrolytes and metabolites can probably be neglected.

Figure S1 shows the sensing performance of self-powered implantable skin-like glucometer under different forces $(14,22,31$, and $40 \mathrm{~N})$. The applied forces have the same frequency of $1.0 \mathrm{~Hz}$. Under the force of $14 \mathrm{~N}$, the outputting piezoelectric voltage of the device against 0 , $0.024,0.045,0.076$, and $0.119 \mathrm{~g} \mathrm{~L}^{-1}$ glucose solutions is $0.45,0.39,0.27,0.18$, and $0.09 \mathrm{~V}$, respectively (Fig. S1a). Under the force of $22 \mathrm{~N}$, the outputting piezoelectric voltage is $0.49,0.42,0.32,0.17$, and $0.11 \mathrm{~V}$, respectively 
(Fig. S1b). Under the force of $31 \mathrm{~N}$, the outputting piezoelectric voltage is $0.60,0.50,0.41,0.29$, and $0.16 \mathrm{~V}$, respectively (Fig. S1c). Under the force of $40 \mathrm{~N}$, the outputting piezoelectric voltage is $0.64,0.56,0.46,0.34$, and $0.22 \mathrm{~V}$, respectively (Fig. S1d). As the applied force increases, the outputting piezoelectric voltage increases (Fig. S1e). Interestingly, as shown in Figure S1f, the response of the device under different applied forces is very close. This feature can facilitate the practical applications of our device.

For showing the flexibility of self-powered implantable skin-like glucometer, the biosensing performance at different bending angles is presented in Fig. S2 [54]. The bending angle is controlled by the moving distance of the stepper motor. Figure S2a shows the outputting piezoelectric voltage of the device against $0.045 \mathrm{~g} \mathrm{~L}^{-1}$ glucose solution at different bending angles. The relationship between outputting piezoelectric voltage and bending angle is shown in Fig. S2b. When the bending angle of the skin-like glucometer is $0^{\circ}, 10^{\circ}, 20^{\circ}$, and $30^{\circ}$, the outputting piezoelectric voltage in $0.045 \mathrm{~g} \mathrm{~L}^{-1}$ glucose solution is $0.27,0.32,0.41$, and $0.45 \mathrm{~V}$, and the voltage in $0 \mathrm{~g} \mathrm{~L}^{-1}$ glucose solution is $0.45,0.49,0.58$, and $0.63 \mathrm{~V}$, respectively. As the bending angle increases, the response decreases (Fig. S2c). It may be attributed to the damage on GOx@ZnO nanowires as the bending angle increases.

The stability of self-powered implantable skin-like glucometer is shown in Fig. S2d. The glucose concentration is $0.045 \mathrm{~g} \mathrm{~L}^{-1}$; the applied force is $21 \mathrm{~N}$; and the bending angle is kept at $20^{\circ}$. After bending for 100 , $200,300,400,500,600,700,800$, and 900 times, the response is $47,51,52,49,49,48,48,53$, and 51 , respectively. These results indicate that the device has high stability.

The working principle of self-powered implantable skinlike glucometer is schematically illustrated in Fig. 5. The working mechanism is based on the coupling between enzymatic reaction and piezo-screening effect of $\mathrm{ZnO}$ nanowires. In pure water, no reactions take place on the surface of GOx@ZnO nanowire, and the surface carrier density of the nanowire is low, as shown in Fig. 5a. Under applied deformation, GOx@ $\mathrm{ZnO}$ nanowire can create piezoelectric potential with weak piezo-screening effect, and the outputting piezoelectric voltage is high (Fig. 5b). When the device is immersed in the glucose solution, the GOx attached on $\mathrm{ZnO}$ nanowire surface reacts with glucose, as shown in Fig. 5c. On the first step, gluconic acid and $\mathrm{H}_{2} \mathrm{O}_{2}$ are generated as the following reaction [55-57]:

Glucose $+\mathrm{H}_{2} \mathrm{O}+\mathrm{O}_{2} \stackrel{\text { GOx }}{\longrightarrow}$ Gluconic acid $+\mathrm{H}_{2} \mathrm{O}_{2}$

It has been reported that $\mathrm{H}_{2} \mathrm{O}_{2}$ can transfer electrons to $\mathrm{ZnO}$ nanowire and produce hydrogen ions as the following decomposition reaction [58]:

$\mathrm{H}_{2} \mathrm{O}_{2} \rightarrow 2 \mathrm{H}^{+}+\mathrm{O}_{2}+2 \mathrm{e}^{-}$ (a)
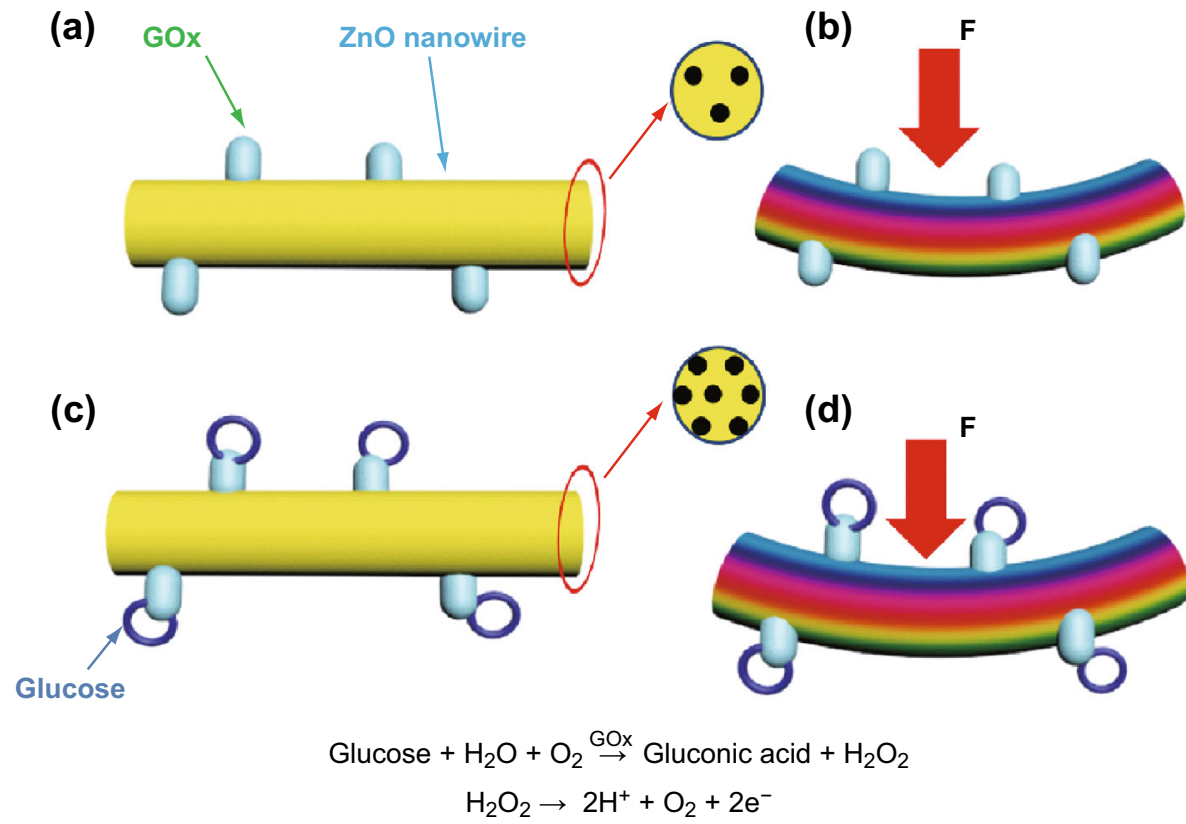

Fig. 5 Working mechanism of self-powered implantable skin-like glucometer. a GOx/ZnO nanowires in pure water without applied deformation. b The piezoelectric output of GOx/ZnO nanowires in pure water under applied deformation. c GOx/ZnO nanowires in glucose aqueous solution without applied deformation. $\mathbf{d}$ The piezoelectric output of GOx/ZnO nanowires in glucose aqueous solution under applied deformation 
In this process, electrons are absorbed on the surface of the nanowire, which can increase the surface carrier density [59]. And $\mathrm{H}^{+}$ions can also be absorbed on the surface of the nanowires, acting as extra carriers [14, 55]. Under applied deformation, the outputting piezoelectric voltage of the nanowire decreases due to the strong piezo-screening effect from the large amount of $\mathrm{H}^{+}$and $\mathrm{e}^{-}$on the surface of the nanowire (Fig. 5d).

To further confirm the mechanism, the response of the device against $\mathrm{H}_{2} \mathrm{O}_{2}$ and $\mathrm{CH}_{3} \mathrm{COOH}\left(\mathrm{H}^{+}\right)$solutions has been tested as shown in Fig. S3. As the concentration of $\mathrm{H}_{2} \mathrm{O}_{2}$ is $0,0.024,0.045,0.076$, and $0.119 \mathrm{~g} \mathrm{~L}^{-1}$, the outputting piezoelectric voltage of the device is about 0.46 , $0.29,0.19,0.13$, and $0.07 \mathrm{~V}$ (Fig. S3a), and the response is about $0,55,133,258$, and 505 (Fig. S3c), respectively. As the concentration of $\mathrm{CH}_{3} \mathrm{COOH}$ is $0,0.024,0.045,0.076$, and $0.119 \mathrm{~g} \mathrm{~L}^{-1}$, the outputting piezoelectric voltage of the device is about $0.601,0.479,0.435,0.358$, and $0.301 \mathrm{~V}$ (Fig. S3b), and the response is about 0, 25, 38, 67, and 99 (Fig. S3d), respectively. It can be seen that the outputting piezoelectric voltage of the device decreases with increasing concentration of $\mathrm{H}_{2} \mathrm{O}_{2}$ or $\mathrm{H}^{+}$. To easily understand all the above experimental results and compare them, Table S1 lists the information.

Figure 6 shows the practical application of self-powered implantable skin-like glucometer implanted in a mouse body for detecting blood glucose concentration without any external electricity power source [60-62]. The device is implanted into the mouse body by surgery process. After that, a constant force of $4 \mathrm{~N}$ controlled by a programming motor is applied on the device in the soft mouse abdomen (Fig. 6a). As the mouse is in anesthesia and cannot have movement, the motor is used to provide force to drive the device. The stepper motor keeps providing constant force (both the frequency and magnitude kept constant) on the device throughout the whole measurement. Thus, the applied strain of the device before and after glucose injection keeps the same. The response of the device is presented in Fig. 6b, c. It was shown that our devices are still able to respond sensitively to the changes in glucose concentration in the biological environment. Without the injection of glucose solution, the blood glucose concentration of mouse is $0.756 \mathrm{~g} \mathrm{~L}^{-1}$ (measured by a commercial blood glucometer), and the outputting piezoelectric voltage is around $0.16 \mathrm{~V}$. After injecting $0.045 \mathrm{~g} \mathrm{~L}^{-1}$ glucose aqueous solution ( $5 \mathrm{~mL}$ ) into the mouse abdomen, the outputting piezoelectric voltage decreases to $0.075 \mathrm{~V}$. The commercial glucometer shows that the blood glucose concentration of mouse is $0.792 \mathrm{~g} \mathrm{~L}^{-1}$. These results can simply and roughly demonstrate that our device can work inside the mouse body and detect changes in blood glucose concentration.

(a)

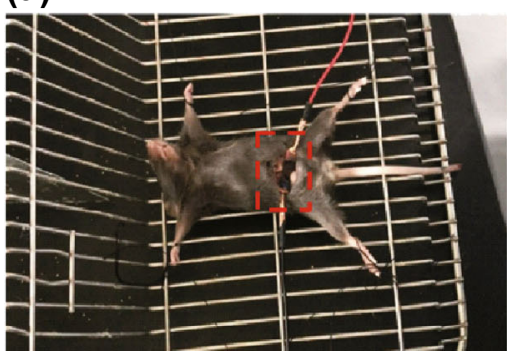

(b)

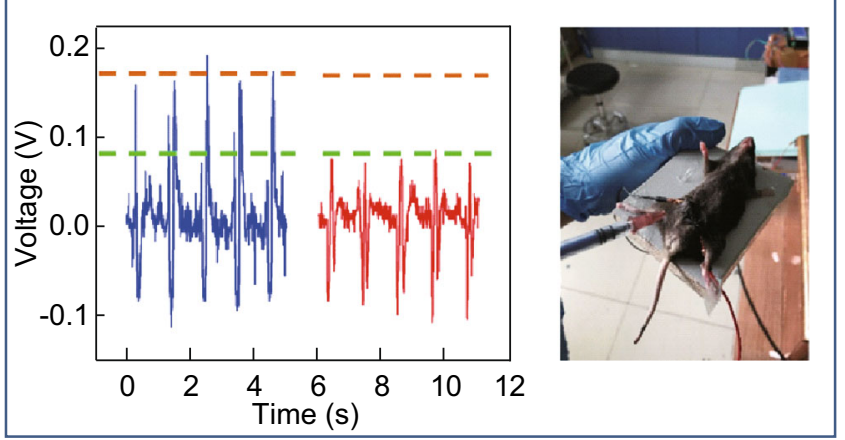

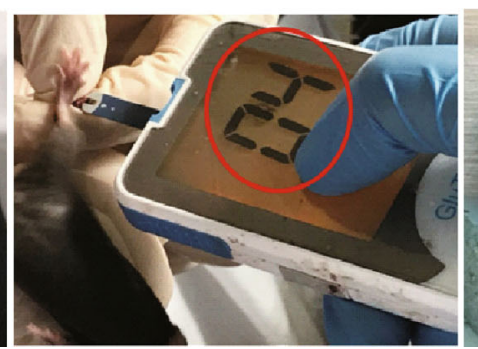

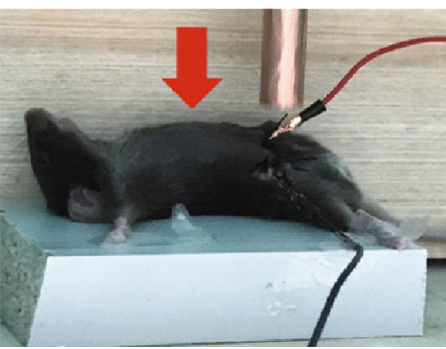

(c)

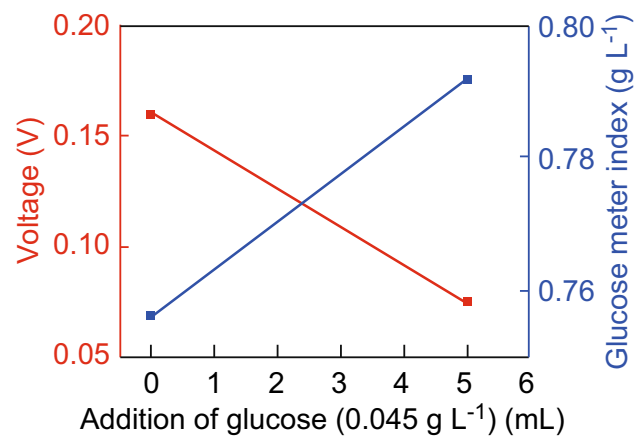

Fig. 6 Practical application of self-powered implantable skin-like glucometer implanted in a mouse body for detecting blood glucose concentration without any external electricity power source. a Implanting the device into the mouse and measurement set. b The outputting piezoelectric voltage changes before and after injection of glucose solution. $\mathbf{c}$ The outputting piezoelectric voltage and glucose meter index of the device before and after the injection of glucose solution 
Our device can harvest tiny mechanical energy of body movement, such as finger pressing or arm flexing. These motions can easily provide enough force (several tens of newton) for driving the device. It should be noted that the device needs to work during the moment of movement and cannot provide its own energy during sleep or rest. In our experiment, the piezoelectric voltage of the device inside the mouse body needs to be tested by external voltmeter. And the device needs to be driven by external applied deformation. Further work can be focused on integrating the signal processor, wireless signal emitter, and mechanical unit with the device, and the whole system can work independently inside the body.

It should also be pointed that the device is still invasive since a "surgery" process is conducted to implant this device into a mouse under the skin, which is against the future demand of noninvasive glucose monitoring. Thus, there are two development direction of the device in the future. One is that the device will detect body fluid outside the skin (tear, saliva, and so on) by improving performance. The working range of our device $\left(0.024-0.119 \mathrm{~g} \mathrm{~L}^{-1}\right)$ is below the concentration found in blood $\left(0.36-5.4 \mathrm{~g} \mathrm{~L}^{-1}\right)$. By considering the size of the device, its biocompatibility and problems with long-term usage of enzymatic sensors, we could probably fabricate a contact lenses glucose sensor (concentration in tears $0.018-0.108 \mathrm{~g} \mathrm{~L}^{-1}$ ) in the future study [17]. The other one is that the overall size of the sensor system will be integrated into the micro-nano-level, and it can be implanted into the human body through a simple method without surgery. It should also be noted that the Kapton film is relatively thick, and this Kapton film cannot be conformably implanted inside the body. The material system and the device structure have not reached the level for practical application. In the future work, we will print the device on much conformable substrates, such as PDMS.

\section{Conclusion}

In summary, we developed a flexible self-powered implantable skin-like glucometer for real-time monitoring blood glucose level in body for the prophylaxis and treatment of diabetes. The working mechanism is based on the piezo-enzymatic-reaction coupling effect of GOx@ $\mathrm{ZnO}$ nanowire arrays. Under applied force, the device can convert the mechanical energy into piezoelectric impulse, which is significantly influenced by blood glucose concentration. The outputting piezoelectric voltage acts as both the biosensing signal and electricity power. A practical application of the device implanted inside mouse body to real-time monitor the blood glucose concentration has been demonstrated. Our work can probably provide a new path for diabetes diagnosis.

Acknowledgements This work was supported by the National Natural Science Foundation of China (11674048), the Fundamental Research Funds for the Central Universities (N160502002), and Liaoning BaiQianWan Talents Program (2014921017).

Open Access This article is distributed under the terms of the Creative Commons Attribution 4.0 International License (http://crea tivecommons.org/licenses/by/4.0/), which permits unrestricted use, distribution, and reproduction in any medium, provided you give appropriate credit to the original author(s) and the source, provide a link to the Creative Commons license, and indicate if changes were made.

\section{References}

1. K. Alberti, P.Z. Zimmet, W.H.O. Consultation, Definition, diagnosis and classification of diabetes mellitus and its complications part 1: diagnosis and classification of diabetes mellitus-provisional report of a who consultation. Diabet. Med. 15(7), 539-553 (1998). https://doi.org/10.1002/(sici)1096-9136(199807)15: 7<539:aid-dia668>3.0.co;2-s

2. S. Wild, G. Roglic, A. Green, R. Sicree, H. King, Global prevalence of diabetes-estimates for the year 2000 and projections for 2030. Diabetes Care 27(5), 1047-1053 (2004). https:// doi.org/10.2337/diacare.27.5.1047

3. A. Gani, A.V. Gribok, S. Rajaraman, W.K. Ward, J. Reifman, Predicting subcutaneous glucose concentration in humans: datadriven glucose modeling. IEEE Trans. Biomed. Eng. 56(2), 246-254 (2009). https://doi.org/10.1109/tbme.2008.2005937

4. D. Son, J. Lee, S. Qiao, R. Ghaffari, J. Kim et al., Multifunctional wearable devices for diagnosis and therapy of movement disorders. Nat. Nanotechnol. 9(5), 397-404 (2014). https://doi.org/10. 1038/nnano.2014.38

5. Y.J. Li, C.C. Lu, W.L. Tsai, M.H. Tai, An intra-oral drug delivery system design for painless, long-term and continuous drug release. Sens. Actuator B Chem. 227, 573-582 (2016). https://doi. org/10.1016/j.snb.2015.12.081

6. T.Q. Trung, N.E. Lee, Flexible and stretchable physical sensor integrated platforms for wearable human-activity monitoring and personal healthcare. Adv. Mater. 28(22), 4338-4372 (2016). https://doi.org/10.1002/adma.201504244

7. N.Q. Luo, W.X. Dai, C.L. Li, Z.Q. Zhou, L.Y. Lu, C.C.Y. Poon, S.C. Chen, Y.T. Zhang, N. Zhao, Flexible piezoresistive sensor patch enabling ultralow power cuffless blood pressure measurement. Adv. Funct. Mater. 26(8), 1178-1187 (2016). https://doi. org/10.1002/adfm.201504560

8. N.M. Iverson, P.W. Barone, M. Shandell, L.J. Trudel, S. Sen et al., In vivo biosensing via tissue-localizable near-infrared-fluorescent single-walled carbon nanotubes. Nat. Nanotechnol. 8(11), 873-880 (2013). https://doi.org/10.1038/nnano.2013.222

9. D. Khodagholy, J.N. Gelinas, T. Thesen, W. Doyle, O. Devinsky, G.G. Malliaras, G. Buzsaki, Neurogrid: recording action potentials from the surface of the brain. Nat. Neurosci. 18(2), 310-315 (2015). https://doi.org/10.1038/nn.3905

10. J. Kim, P. Gutruf, A.M. Chiarelli, S.Y. Heo, K. Cho et al., Miniaturized battery-free wireless systems for wearable pulse oximetry. Adv. Funct. Mater. 27(1), 1604373 (2017). https://doi. org/10.1002/adfm.201604373 
11. I. Ilhan, I. Yildiz, M. Kayrak, Development of a wireless blood pressure measuring device with smart mobile device. Comput. Methods Programs Biomed. 125, 94-102 (2016). https://doi.org/ 10.1016/j.cmpb.2015.11.003

12. M. Staples, K. Daniel, M.J. Cima, R. Langer, Application of micro- and nano-electromechanical devices to drug delivery. Pharmacol. Res. 23(5), 847-863 (2006). https://doi.org/10.1007/ s11095-006-9906-4

13. G. Sehra, M. Cole, J.W. Gardner, Miniature taste sensing system based on dual sh-saw sensor device: an electronic tongue. Sens. Actuator B Chem. 103(1-2), 233-239 (2004). https://doi.org/10. 1016/j.snb.2004.04.055

14. X.Y. Xue, Z. Qu, Y.M. Fu, B.W. Yu, L.L. Xing, Y. Zhang, Selfpowered electronic-skin for detecting glucose level in body fluid basing on piezo-enzymatic-reaction coupling process. Nano Energy 26, 148-156 (2016). https://doi.org/10.1016/j.nanoen. 2016.05.021

15. Y. Xiang, Y. Lu, Using personal glucose meters and functional DNA sensors to quantify a variety of analytical targets. Nat. Chem. 3(9), 697-703 (2011). https://doi.org/10.1038/nchem.1092

16. G.S. Wilson, R. Gifford, Biosensors for real-time in vivo measurements. Biosens. Bioelectron. 20(12), 2388-2403 (2005). https://doi.org/10.1016/j.bios.2004.12.003

17. E.W. Nery, M. Kundys, P.S. Jelen, M. Jonsson-Niedziolka, Electrochemical glucose sensing: is there still room for improvement? Anal. Chem. 88(23), 11271-11282 (2016). https:// doi.org/10.1021/acs.analchem.6b03151

18. R. Gifford, Continuous glucose monitoring: 40 years, what we've learned and what's next. ChemPhysChem 14(10), 2032-2044 (2013). https://doi.org/10.1002/cphc.201300172

19. Y. Zhang, X.Q. Yan, Y. Yang, Y.H. Huang, Q.L. Liao, J.J. Qi, Scanning probe study on the piezotronic effect in zno nanomaterials and nanodevices. Adv. Mater. 24(34), 4647-4655 (2012). https://doi.org/10.1002/adma.201104382

20. Y. Zhang, Y. Yang, Y.S. Gu, X.Q. Yan, Q.L. Liao, P.F. Li, Z. Zhang, Z.Z. Wang, Performance and service behavior in 1-d nanostructured energy conversion devices. Nano Energy 14, 30-48 (2015). https://doi.org/10.1016/j.nanoen.2014.12.039

21. M.Y. Ma, Q.L. Liao, G.J. Zhang, Z. Zhang, Q.J. Liang, Y. Zhang, Self-recovering triboelectric nanogenerator as active multifunctional sensors. Adv. Funct. Mater. 25(41), 6489-6494 (2015). https://doi.org/10.1002/adfm.201503180

22. M.Y. Ma, Z. Zhang, Q.L. Liao, F. Yi, L.H. Han, G.J. Zhang, S. Liu, X.Q. Liao, Y. Zhang, Self-powered artificial electronic skin for high-resolution pressure sensing. Nano Energy 32, 389-396 (2017). https://doi.org/10.1016/j.nanoen.2017.01.004

23. Z.L. Wang, The new field of nanopiezotronics. Mater. Today 10(5), 20-28 (2007). https://doi.org/10.1016/s1369-7021(07)700 76-7

24. S. Lee, R. Hinchet, Y. Lee, Y. Yang, Z.H. Lin, G. Ardila, L. Montes, M. Mouis, Z.L. Wang, Ultrathin nanogenerators as selfpowered/active skin sensors for tracking eye ball motion. Adv. Funct. Mater. 24(8), 1163-1168 (2014). https://doi.org/10.1002/ adfm. 201301971

25. H.L. Zhang, Y. Yang, T.C. Hou, Y.J. Su, C.G. Hu, Z.L. Wang, Triboelectric nanogenerator built inside clothes for self-powered glucose biosensors. Nano Energy 2(5), 1019-1024 (2013). https:// doi.org/10.1016/j.nanoen.2013.03.024

26. I. You, B. Kim, J. Park, K. Koh, S. Shin, S. Jung, U. Jeong, Stretchable e-skin apexcardiogram sensor. Adv. Mater. 28(30), 6359-6364 (2016). https://doi.org/10.1002/adma.201600720

27. Y. Zhang, Z. Kang, X.Q. Yan, Q.L. Liao, Zno nanostructures in enzyme biosensors. Sci. China Mater. 58(1), 60-76 (2015). https://doi.org/10.1007/s40843-015-0017-6

28. P. Singh, S.K. Pandey, J. Singh, S. Srivastava, S. Sachan, S.K. Singh, Biomedical perspective of electrochemical nanobiosensor.
Nano-Micro Lett. 8, 193 (2016). https://doi.org/10.1007/s40820015-0077-x

29. H.N. Si, Z. Kang, Q.L. Liao, Z. Zhang, X.M. Zhang, L. Wang, Y. Zhang, Design and tailoring of patterned $\mathrm{ZnO}$ nanostructures for energy conversion applications. Sci. China Mater. 60(9), 793-810 (2017). https://doi.org/10.1007/s40843-017-9105-3

30. S.S. Ma, J.J. Xue, Y.M. Zhou, Z.W. Zhang, Photochemical synthesis of $\mathrm{ZnO} / \mathrm{Ag}_{2} \mathrm{O}$ heterostructures with enhanced ultraviolet and visible photocatalytic activity. J. Mater. Chem. A 2(20), 7272-7280 (2014). https://doi.org/10.1039/c4ta00464g

31. L. Zhang, S. Bai, C. Su, Y.B. Zheng, Y. Qin, C. Xu, Z.L. Wang, A high-reliability kevlar fiber-zno nanowires hybrid nanogenerator and its application on self-powered uv detection. Adv. Funct. Mater. 25(36), 5794-5798 (2015). https://doi.org/10.1002/adfm. 201502646

32. M. Kevin, Y.H. Fou, A.S.W. Wong, G.W. Ho, A novel maskless approach towards aligned, density modulated and multi-junction zno nanowires for enhanced surface area and light trapping solar cells. Nanotechnology 21(31), 9 (2010). https://doi.org/10.1088/ $0957-4484 / 21 / 31 / 315602$

33. Y.M. Sung, K. Noh, W.C. Kwak, T.G. Kim, Enhanced glucose detection using enzyme-immobilized zno/zns core/sheath nanowires. Sens. Actuator B Chem. 161(1), 453-459 (2012). https:// doi.org/10.1016/j.snb.2011.10.061

34. J.C. Yu, Y.J. Zhang, S.Q. Liu, Enzymatic reactivity of glucose oxidase confined in nanochannels. Biosens. Bioelectron. 55, 307-312 (2014). https://doi.org/10.1016/j.bios.2013.12.042

35. H.X. He, Y.M. Fu, W.L. Zang, Q. Wang, L.L. Xing, Y. Zhang, X.Y. Xue, A flexible self-powered t-zno/pvdf/fabric electronicskin with multi functions of tactile-perception, atmosphere-detection and self-clean. Nano Energy 31, 37-48 (2017). https://doi. org/10.1016/j.nanoen.2016.11.020

36. Y. Sun, S.P. Lacour, R.A. Brooks, N. Rushton, J. Fawcett, R.E. Cameron, Assessment of the biocompatibility of photosensitive polyimide for implantable medical device use. J. Biomed. Mater. Res. A 90A(3), 648-655 (2009). https://doi.org/10.1002/jbm.a. 32125

37. M. Niinomi, Fatigue performance and cyto-toxicity of low rigidity titanium alloy, Ti-29Nb-13Ta-4.6Zr. Biomaterials 24(16), 26732683 (2003). https://doi.org/10.1016/s0142-9612(03)00069-3

38. A. Wei, L.H. Pan, W. Huang, Recent progress in the zno nanostructure-based sensors. Mater. Sci. Eng. B Adv. Funct. Solid State Mater. 176(18), 1409-1421 (2011). https://doi.org/10. 1016/j.mseb.2011.09.005

39. M. Geetha, A.K. Singh, R. Asokamani, A.K. Gogia, Ti based biomaterials, the ultimate choice for orthopaedic implants-a review. Prog. Mater. Sci. 54(3), 397-425 (2009). https://doi.org/ 10.1016/j.pmatsci.2008.06.004

40. X. Chen, J.G. Patil, S.H.L. Lok, O.L. Kon, Human liver-derived cells stably modified for regulated proinsulin secretion function as bioimplants in vivo. J. Gene Med. 4(4), 447-458 (2002). https://doi.org/10.1002/jgm.263

41. Y.J. Su, X.N. Wen, G. Zhu, J. Yang, J. Chen, P. Bai, Z.M. Wu, Y.D. Jiang, Z.L. Wang, Hybrid triboelectric nanogenerator for harvesting water wave energy and as a self-powered distress signal emitter. Nano Energy 9, 186-195 (2014). https://doi.org/ 10.1016/j.nanoen.2014.07.006

42. M.Y. Choi, D. Choi, M.J. Jin, I. Kim, S.H. Kim, J.Y. Choi, S.Y. Lee, J.M. Kim, S.W. Kim, Mechanically powered transparent flexible charge-generating nanodevices with piezoelectric $\mathrm{ZnO}$ nanorods. Adv. Mater. 21(21), 2185-2189 (2009). https://doi.org/ 10.1002/adma.200803605

43. X. Liu, P. Lin, X.Q. Yan, Z. Kang, Y.G. Zhao, Y. Lei, C.B. Li, H.W. Du, Y. Zhang, Enzyme-coated single $\mathrm{ZnO}$ nanowire fet biosensor for detection of uric acid. Sens. Actuator B Chem. 176, 22-27 (2013). https://doi.org/10.1016/j.snb.2012.08.043 
44. L. Guo, Y.L. Ji, H.B. Xu, P. Simon, Z.Y. Wu, Regularly shaped, single-crystalline zno nanorods with wurtzite structure. J. Am. Chem. Soc. 124(50), 14864-14865 (2002). https://doi.org/10. $1021 / \mathrm{ja} 027947 \mathrm{~g}$

45. J.Y. Lao, J.Y. Huang, D.Z. Wang, Z.F. Ren, ZnO nanobridges and nanonails. Nano Lett. 3(2), 235-238 (2003). https://doi.org/ $10.1021 / \mathrm{nl} 025884 \mathrm{u}$

46. H.Y. Zhao, X.Y. Guo, Y.Y. Wang, X.X. Duan, H.M. Qu, H. Zhang, D.H. Zhang, W. Pang, Microchip based electrochemicalpiezoelectric integrated multi-mode sensing system for continuous glucose monitoring. Sens. Actuator B Chem. 223, 83-88 (2016). https://doi.org/10.1016/j.snb.2015.09.022

47. J.F. Zang, C.M. Li, X.Q. Cui, J.X. Wang, X.W. Sun, H. Dong, C.Q. Sun, Tailoring zinc oxide nanowires for high performance amperometric glucose sensor. Electroanalysis 19(9), 1008-1014 (2007). https://doi.org/10.1002/elan.200603808

48. J.X. Wang, X.W. Sun, A. Wei, Y. Lei, X.P. Cai, C.M. Li, Z.L. Dong, Zinc oxide nanocomb biosensor for glucose detection. Appl. Phys. Lett. 88, 233106 (2006). https://doi.org/10.1063/1. 2210078

49. W.L. Zang, Y.X. Nie, D. Zhu, P. Deng, L.L. Xing, X.Y. Xue, Core-shell $\mathrm{In}_{2} \mathrm{O}_{3} / \mathrm{ZnO}$ nanoarray nanogenerator as a self-powered active gas sensor with high $\mathrm{H}_{2} \mathrm{~S}$ sensitivity and selectivity at room temperature. J. Phys. Chem. C 118(17), 9209-9216 (2014). https://doi.org/10.1021/jp500516t

50. Y.Y. Zhao, Y.M. Fu, P.L. Wang, L.L. Xing, X.Y. Xue, Highly stable piezo-immunoglobulin-biosensing of a $\mathrm{SiO}_{2} / \mathrm{ZnO}$ nanogenerator as a self-powered/active biosensor arising from the field effect influenced piezoelectric screening effect. Nanoscale 7(5), 1904-1911 (2015). https://doi.org/10.1039/c4nr06461e

51. T. Kong, Y. Chen, Y.P. Ye, K. Zhang, Z.X. Wang, X.P. Wang, An amperometric glucose biosensor based on the immobilization of glucose oxidase on the $\mathrm{ZnO}$ nanotubes. Sens. Actuator B Chem. 138(1), 344-350 (2009). https://doi.org/10.1016/j.snb. 2009.01.002

52. S.Q. Liu, H.X. Ju, Reagentless glucose biosensor based on direct electron transfer of glucose oxidase immobilized on colloidal gold modified carbon paste electrode. Biosens. Bioelectron. 19(3), 177-183 (2003). https://doi.org/10.1016/s0956-5663(03)00172-6

53. S.N. Sarangi, S. Nozaki, S.N. Sahu, ZnO nanorod-based nonenzymatic optical glucose biosensor. J. Biomed. Nanotechnol. 11(6), 988-996 (2015). https://doi.org/10.1166/jbn.2015.2048
54. M.D. Bartlett, E.J. Markvicka, C. Majidi, Rapid fabrication of soft, multilayered electronics for wearable biomonitoring. Adv. Funct. Mater. 26(46), 8496-8504 (2016). https://doi.org/10.1002/ adfm. 201602733

55. R.M. Yu, C.F. Pan, J. Chen, G. Zhu, Z.L. Wang, Enhanced performance of a zno nanowire-based self-powered glucose sensor by piezotronic effect. Adv. Funct. Mater. 23(47), 5868-5874 (2013). https://doi.org/10.1002/adfm.201300593

56. M.M. Rahman, A.J.S. Ahammad, J.H. Jin, S.J. Ahn, J.J. Lee, A comprehensive review of glucose biosensors based on nanostructured metal-oxides. Sensors 10(5), 4855-4886 (2010). https:// doi.org/10.3390/s100504855

57. F.F. Zhang, X.L. Wang, S.Y. Ai, Z.D. Sun, Q. Wan, Z.Q. Zhu, Y.Z. Xian, L.T. Jin, K. Yamamoto, Immobilization of uricase on zno nanorods for a reagentless uric acid biosensor. Anal. Chim. Acta 519(2), 155-160 (2004). https://doi.org/10.1016/j.aca.2004. 05.070

58. M. Ahmad, C.F. Pan, Z.X. Luo, J. Zhu, A single ZnO nanofiberbased highly sensitive amperometric glucose biosensor. J. Phys. Chem. C 114(20), 9308-9313 (2010). https://doi.org/10.1021/ jp102505g

59. G.A. Zhu, R.S. Yang, S.H. Wang, Z.L. Wang, Flexible highoutput nanogenerator based on lateral $\mathrm{ZnO}$ nanowire array. Nano Lett. 10(8), 3151-3155 (2010). https://doi.org/10.1021/nl101973h

60. Y. Tanaka, H. Fujita, Fluid driving system for a micropump by differentiating ips cells into cardiomyocytes on a tent-like structure. Sens. Actuator B Chem. 210, 267-272 (2015). https:// doi.org/10.1016/j.snb.2014.12.069

61. W. Farris, S. Mansourian, Y. Chang, L. Lindsley, E.A. Eckman et al., Insulin-degrading enzyme regulates the levels of insulin, amyloid beta-protein, and the beta-amyloid precursor protein intracellular domain in vivo. Proc. Natl. Acad. Sci. USA 100(7), 4162-4167 (2003). https://doi.org/10.1073/pnas.0230450100

62. R.D. Jayant, M.J. McShane, R. Srivastava, In vitro and in vivo evaluation of anti-inflammatory agents using nanoengineered alginate carriers: Towards localized implant inflammation suppression. Int. J. Pharm. 403(1-2), 268-275 (2011). https://doi.org/ 10.1016/j.ijpharm.2010.10.035 